\title{
The role of intraoperative MRI in resective epilepsy surgery for peri-eloquent cortex cortical dysplasias and heterotopias in pediatric patients
}

\author{
Matthew F. Sacino, BS, ${ }^{1}$ Cheng-Ying Ho, MD, PhD, ${ }^{3}$ Jonathan Murnick, MD, PhD, ${ }^{2}$ \\ Robert F. Keating, MD, ${ }^{1}$ William D. Gaillard, MD, ${ }^{4}$ and Chima O. Oluigbo, MD ${ }^{1}$ \\ Departments of ${ }^{1}$ Neurosurgery, ${ }^{2}$ Neuroradiology, ${ }^{3}$ Neuropathology, and ${ }^{4}$ Neurology, Children's National Medical Center, \\ Washington, DC
}

OBJECTIVE Previous studies have demonstrated that an important factor in seizure freedom following surgery for lesional epilepsy in the peri-eloquent cortex is completeness of resection. However, aggressive resection of epileptic tissue localized to this region must be balanced with the competing objective of retaining postoperative neurological functioning. The objective of this study was to investigate the role of intraoperative MRI (iMRI) as a complement to existing epilepsy protocol techniques and to compare rates of seizure freedom and neurological deficit in pediatric patients undergoing resection of peri-eloquent lesions.

METHODS The authors retrospectively reviewed the medical records of pediatric patients who underwent resection of focal cortical dysplasia (FCD) or heterotopia localized to eloquent cortex regions at the Children's National Health System between March 2005 and August 2015. Patients were grouped into two categories depending on whether they underwent conventional resection $(n=18)$ or iMRI-assisted resection $(n=11)$. Patient records were reviewed for factors including demographics, length of hospitalization, postoperative seizure freedom, postoperative neurological deficit, and need for reoperation. Postsurgical seizure outcome was assessed at the last postoperative follow-up evaluation using the Engel Epilepsy Surgery Outcome Scale.

RESULTS At the time of the last postoperative follow-up examination, $9(82 \%)$ of the 11 patients in the iMRI resection group were seizure free (Engel Class I), compared with $7(39 \%)$ of the 18 patients in the control resection group $(p=$ 0.05). Ten (91\%) of the 11 patients in the iMRI cohort achieved gross-total resection (GTR), compared with 8 (44\%) of 18 patients in the conventional resection cohort $(p=0.02)$. One patient in the iMRI-assisted resection group underwent successful reoperation at a later date for residual dysplasia, compared with 7 patients in the conventional resection cohort (with 2/7 achieving complete resection). Four (36\%) of the patients in the iMRI cohort developed postoperative neurological deficits, compared with 15 patients $(83 \%)$ in the conventional resection cohort $(p=0.02)$.

CONCLUSIONS These results suggest that in comparison with a conventional surgical protocol and technique for resection of epileptic lesions in peri-eloquent cortex, the incorporation of iMRI led to elevated rates of GTR and postoperative seizure freedom. Furthermore, this study suggests that iMRI-assisted surgeries are associated with a reduction in neurological deficits due to intraoperative damage of eloquent cortex.

http://thejns.org/doi/abs/10.3171/2016.1.FOCUS15538

KEY WORDS intraoperative magnetic resonance imaging; focal cortical dysplasia; epilepsy surgery; pediatric; eloquent cortex; heterotopia

$\mathrm{R}$ ESECTION of focal cortical dysplasia (FCD) and heterotopia localized adjacent to eloquent cortex presents a significant challenge of competing objectives. A balance must be attained between achieving the ultimate goal of complete seizure freedom while minimizing postoperative neurological deficits.
Previous studies have demonstrated that an important factor in achieving postoperative seizure freedom for patients presenting with intractable epilepsy secondary to a lesion adjacent to eloquent cortex is gross-total resection (GTR). ${ }^{9,28}$ Traditionally, aggressive resections in the peri-eloquent regions have been tempered by high rates

ABBREVIATIONS CNHS = Children's National Health System; DTI = diffusion tensor imaging; DWI = diffusion-weighted imaging; ECoG = electrocorticography; FCD = focal cortical dysplasia; GTR = gross-total resection; iMRI = intraoperative MRI; MEP = motor evoked potential; SEM = standard error of the mean; SSEP = somatosensory evoked potential. 
of neurological deficit, and seizure-free rates in pediatric patients following surgery range from $43 \%$ to $64 \%$. $1,2,8,24$ Intraoperative identification of the extent of resection with compensation for brain shift is a critical factor in perieloquent resections, which has been shown to be aided by intraoperative MRI (iMRI). ${ }^{20,22}$ iMRI has previously been shown to aid surgeries of eloquent cortex gliomas, leading to increased rates of complete resection and fewer postoperative deficits. ${ }^{13,19,30,31}$ Furthermore, iMRI has been successfully integrated into pediatric cases and has been shown to aid in intraoperative evaluation of the extent of resection in epilepsy surgery. $3,4,21,32$ Hence, we sought to adapt this technology in resective epilepsy surgery for epileptogenic lesions located adjacent to eloquent cortex. The current study is a comparative analysis of postoperative seizure and neurological outcomes in patients undergoing resection of FCD and heterotopias adjacent to eloquent cortex using iMRI-assisted versus conventional resective techniques.

\section{Methods \\ Patient Population}

We retrospectively reviewed the medical records and MR images of 29 pediatric patients undergoing surgery at Children's National Health System (CNHS) for pharmacoresistant epilepsy secondary to FCD localized adjacent to eloquent cortex (motor, sensory, visual, and speech) who met inclusion criteria. Resections between March 2005 and April $2013(\mathrm{n}=18)$ were performed via a conventional microsurgical technique with the aid of neuronavigation (Stealth Station navigation system, Medtronic-Sofamor Danek), neurophysiological monitoring, and intraoperative electrocorticography (ECoG), while surgeries conducted between January 2014 and August 2015 $(\mathrm{n}=11)$ were performed with the additional aid of 1.5-T iMRI to the previously used assistive neurotechnologies. Upon approval from the institutional review board, patient records were assessed for demographic data, new postoperative deficits, completion of resection, postoperative seizure outcome, length of surgery, length of hospitalization, and need for reoperation. For conventional resection patients undergoing repeat resection of residual dysplasia, duration of follow-up was extended until the time of repeat surgery.

\section{Eloquent Cortex}

Eloquent cortex is defined as areas of brain that are responsible for highly critical and complex functions that the brain cannot easily compensate for, including areas of brain that subserve motor, sensory, language, or visual function. Eloquent cortex was defined by 4 parameters. All patients presented with structural lesions on preoperative MRI indicating proximity to eloquent regions. Functional MRI (fMRI) with motor and language paradigms was performed as indicated in patients who were old enough to cooperate with this investigation. Patients with peri-rolandic lesions underwent intraoperative brain mapping with electrophysiological phase-reversal assessment to identify the central sulcus. Additionally, 2 patients in the conventional resection cohort underwent extraop- erative brain mapping through grids that had been placed intraoperatively.

\section{Surgical Procedure}

We previously described a conventional microsurgical technique for resection of FCD at our institution. ${ }^{23}$ In the iMRI cohort, ECoG was performed in 9 of the 11 resections but was not used in patients with deep lesions that posed a significant risk of vascular injury. Intraoperative neurophysiological monitoring of somatosensory evoked potentials (SSEPs) and motor evoked potentials (MEPs) was used in all cases. Standard microsurgical resection was conducted until the surgeon believed that a complete resection had been performed. The patient was then prepared and transferred to the dedicated 1.5-T iMRI suite (Greenline Achieva Nova Dual, Philips Medical Systems). The intraoperative sequences taken in the iMRI cohort included 1) a T1-weighted 3D fast field echo (TE $3.2 \mathrm{msec}$, TR $6.7 \mathrm{msec}$, matrix $240 \times 240$, FOV $300 \mathrm{~mm}$, slice thickness $1.6 \mathrm{~mm}$, slab $34 \mathrm{~cm}$ ) reformatted into orthogonal planes; 2) T2-weighted turbo spin-echo (TE $100 \mathrm{msec}$, TR $4019 \mathrm{msec}$, matrix $296 \times 225$, FOV $280 \mathrm{~mm}$, slice thickness $3 \mathrm{~mm}$ ); and 3) a diffusion-weighted imaging (DWI) sequence (TE $103 \mathrm{msec}$, TR $5096 \mathrm{msec}$, matrix $152 \times 106$, FOV $230 \mathrm{~mm}$, slice thickness $4 \mathrm{~mm}$ ). Images were independently viewed and analyzed by the attending neurosurgeon and neuroradiologist and discussed jointly. In cases of residual dysplasia, the patient was returned to surgery for further reexploration at the same operative session.

\section{Duration of Operative Event}

Duration of the operative event was defined as the recorded time of entry into the operating room suite until exit. Hence, in addition to surgical time, this encompassed time for anesthesia, placement of peripheral lines, preparing the patient, acquisition of all MR images, and anesthesia recovery. These extrasurgical activities accounted for 2-3 hours of the duration on average. We believed it was relevant to include these other (extraoperative) components because they are a reflection of the complexity of coordination of care and are therefore indirect costs in undertaking this type of procedure.

\section{Neurological and Seizure Outcomes}

Neurological deficits were retrospectively assessed at discharge, the first postoperative visit, and at the last postoperative visit. Postoperative neurological function was compared with the preoperative baseline level and evaluated for alterations. Transient deficit was defined as complete recovery to preoperative baseline by the last postoperative follow-up evaluation. Permanent deficit was defined as persistence of the deficit at the last postoperative follow-up. Mild deficits were defined as nondisabling hemiparesis or hemianesthesia. Severe deficits were defined as disabling hemiparesis or hemianesthesia. Postsurgical seizure outcome was assessed utilizing the Engel Epilepsy Surgery Outcome Scale ${ }^{10}$ at the last postoperative follow-up visit.

\section{Statistical Analysis}

Clinical and demographic statistics were recorded as 
means \pm standard errors of the mean (SEM), and duration of follow-up was reported as median. Statistical analysis of continuous variables between the independent cohorts was performed via the nonparametric Mann-Whitney Utest. The Fischer exact test was used to assess categorical variables between groups. All tests performed were 2 -tailed, and a $\mathrm{p}$ value $<0.05$ was considered statistically significant.

\section{Results \\ Patient Population}

Between March 2005 and August 2015, 29 consecutive patients underwent resection of FCD and heterotopias localized adjacent to eloquent cortex at CNHS. Eighteen patients (13 males and 5 females) underwent resection utilizing conventional epilepsy protocol and techniques, and 11 patients ( 3 males and 8 females) underwent iMRIassisted resection. There were no statistically significant differences between the two groups for the variables of mean age at surgery, mean duration of seizures, or mean age at seizure onset (Table 1). The median duration of follow-up was 5.72 months (range $0.46-10.61$ months) for the iMRI-assisted cohort and 17.75 months (range 0.13-71.85 months) for the conventional cohort. Patients in the iMRI cohort had a shorter mean length of stay than those in the conventional cohort $(3.8 \pm 0.4$ days vs $4.9 \pm 0.5$ days, respectively), although this difference was not statistically significant $(p=0.08)$.

Demographics and surgical data are illustrated in Table 2 for patients in the conventional resection cohort, and in Table 3 for patients in the iMRI-assisted resection cohort. Pathohistological analysis of patients undergoing iMRIassisted resection revealed 2 Type I FCDs, 5 Type IIA FCDs, 2 Type IIB FCDs, and 2 FCDs of undetermined histology. In the conventional resection cohort there were 6 Type I FCDs, 3 Type IIA FCDs, 7 Type IIB FCDs, and 2 FCDs of undetermined histology.

\section{Intraoperative MRI Surgeries}

As expected, iMRI-resections were statistically longer in duration than conventional resections $(409.5 \pm 44.9$ minutes vs $289.6 \pm 13.9$ minutes, respectively; $p=0.004$ ). Mean total acquisition time per case was $14.7 \pm 2.4$ minutes. In addition to acquisition time, preparation and transport for iMRI accounted for approximately 45-60 minutes. Four of the patients (36\%) returned to the operating suite in the same operative session for further exploration of the resection cavity following the iMRI indication of residual dysplasia, and further resection was performed in 2 (18\%) of these patients (Cases 4 and 9). Further intraoperative exploration confirmed complete resection in 1 patient (Case 2), and further resection was halted in 1 patient (Case 8) due to reduction in evoked potentials.

\section{Resective and Postoperative Seizure Outcomes}

The iMRI cohort achieved a greater rate of GTR of the MRI-indicated dysplastic lesion than the conventional resection group $(\mathrm{p}=0.02)$. Intraoperative 1.5 -T or postoperative 3.0-T MRI confirmed complete resection in 10 (91\%) of the 11 patients undergoing iMRI-assisted FCD resection, whereas postoperative 1.5- or 3.0-T MRI confirmed complete resection in $8(44 \%)$ of the 18 patients in the conventional resection cohort. One patient in the iMRI cohort underwent a further resection at a later date to achieve a GTR, while 7 patients in the conventional FCD resection cohort underwent repeat resections at a later date and 2 of 7 achieved GTRs.

At the time of the last postoperative follow-up evaluation, $9(82 \%)$ of the 11 patients in the iMRI-assisted group achieved complete seizure freedom (Engel Class I) compared with 7 (39\%) of the 18 patients in the conventional resection group ( $\mathrm{p}=0.05 ;$ Fig. 1). The median duration to seizure recurrence in patients from the conventional resection group $(\mathrm{n}=11)$ was 2.23 months postoperatively (ranging from postoperative Day 1 to 28.5 months postoperatively). One patient (Case 8) in the iMRI cohort underwent further reoperation at a later date, achieving complete seizure freedom postoperatively. Seven patients in the conventional resection cohort underwent further surgery at a later date with 2 achieving complete seizure freedom.

\section{Neurological Outcome}

Patients undergoing conventional FCD resection were more likely to develop postoperative neurological deficits than patients undergoing iMRI-assisted resections ( $\mathrm{p}$ $=0.02$; Fig. 2). Four (36\%) of the patients in the iMRI cohort developed transient neurological deficits (resolved by the last postoperative visit) following surgery. Two patients (Cases 4 and 6) developed mild motor deficits, and 2 patients (Cases 5 and 9) reported transient alteration of sensation in the lower extremities. Fifteen $(83 \%)$ of the patients in the conventional resection group developed transient $(n=3)$ or more permanent $(n=12)$ deficits following

TABLE 1. Comparative analysis between cohorts*

\begin{tabular}{lccc}
\hline \multicolumn{1}{c}{ Variable } & $\begin{array}{c}\text { iMRI Incorporated } \\
\text { Resection Group }\end{array}$ & $\begin{array}{c}\text { Conventional Epilepsy Protocol } \\
\text { Resection Group }\end{array}$ & $p$ Value \\
\hline Age at surgery (yrs) & $9.1 \pm 2.0$ & $6.1 \pm 1.1$ & 0.2 \\
\hline Age at seizure onset (yrs) & $3.49 \pm 1.0$ & $2.7 \pm 9.1$ & 0.5 \\
\hline Duration of seizures (mos) & $59.5 \pm 20.0$ & $41.4 \pm 11.3$ & 0.4 \\
\hline Duration of follow-up (mos) & $5.02 \pm 1.2$ & $23.3 \pm 5.0$ & 0.005 \\
\hline Duration of operative event (mins) & $409.5 \pm 44.9$ & $289.6 \pm 13.9$ & 0.004 \\
\hline Postop length of stay (days) & $3.8 \pm 0.4$ & $4.9 \pm 0.5$ & 0.08 \\
\hline * All data given as mean + SEM. & & & \\
\end{tabular}


TABLE 2. Descriptive characteristics for patients undergoing conventional peri-eloquent resections

\begin{tabular}{|c|c|c|c|c|c|c|c|}
\hline Case No. & $\begin{array}{l}\text { Age (yrs), } \\
\text { Sex }\end{array}$ & $\begin{array}{l}\text { Histopathology } \\
\text { (FCD type) }\end{array}$ & Lesion Localization & $\begin{array}{c}\text { Operative } \\
\text { Duration (mins) }\end{array}$ & $\begin{array}{l}\text { Follow-up } \\
\text { (mos) }\end{array}$ & $\begin{array}{l}\text { Engel } \\
\text { Class }\end{array}$ & Primary Postop Morbidity \\
\hline 1 & $0.2, \mathrm{M}$ & IIB & Rt frontal & 213 & 13.0 & IV & Lt hemiparesis \\
\hline 2 & $2.5, \mathrm{M}$ & IIB & Lt frontal & 270 & 2.5 & ॥ & Rt hemiparesis \\
\hline 3 & $11, \mathrm{~F}$ & NS & Lt temporal & 377 & 61.9 & 1 & Rt superior quadranopsia \\
\hline 4 & $0.3, \mathrm{M}$ & $\| A$ & Rt parietal & 355 & 22.5 & III & Lt hemiparesis \\
\hline 5 & $1.5, \mathrm{M}$ & IIB & Rt parietal & 316 & 71.9 & I & Lt hemiparesis \\
\hline 6 & $6, \mathrm{~F}$ & NS & Rt parietal & 218 & 0.6 & III & Lt hemiparesis \\
\hline 7 & $4.5, M$ & $\| A$ & Lt frontal & 263 & 12.0 & III & Rt hemiparesis \\
\hline 8 & $10, M$ & IIB & Rt frontal & 280 & 50.2 & II & Lt hemiparesis \\
\hline 9 & $2.5, \mathrm{~F}$ & IIB & Lt temporal & 239 & 24.0 & 1 & Expressive language deficit \\
\hline 10 & $4, M$ & I & Rt occipital & 288 & 0.1 & I & Exophoria \\
\hline 11 & $3, \mathrm{M}$ & $\| A$ & Lt frontotemporal & 431 & 4.8 & III & Rt hemiparesis \\
\hline 12 & $8, M$ & 1 & Lt temporal & 327 & 7.1 & III & Rt hemiparesis \\
\hline 13 & $13, \mathrm{M}$ & I & Lt frontal & 328 & 9.8 & III & No \\
\hline 14 & $15, F$ & 1 & Lt parietal & 303 & 41.3 & I & Receptive language deficit \\
\hline 15 & $0.2, \mathrm{M}$ & IIB & Rt parietal & 234 & 33.4 & II & Lt hemiparesis \\
\hline 16 & $7.5, \mathrm{~F}$ & I & $\begin{array}{l}\text { Lt frontal transmantle } \\
\text { heterotopia }\end{array}$ & 234 & 10.7 & I & Rt hemiparesis \\
\hline 17 & $6.5, \mathrm{M}$ & I & Rt frontal & 294 & 27.6 & 1 & No \\
\hline 18 & $13.5, \mathrm{M}$ & IIB & Rt frontoparietal & 242 & 25.5 & II & No \\
\hline
\end{tabular}

NS = not specified.

surgery. Nine patients developed permanent motor deficit following surgery; 8 patients developed mild unresolved weakness, and 1 patient developed more severe hemiparesis that persisted despite rehabilitation. There were 2 cases of transient hemiparesis. Of the remaining patients who developed postoperative deficits, there were 2 cases of permanent visual field deficits, 1 case of transient language deficit, and 1 case of permanent language deficit.

\section{Illustrative Case}

A 20-month-old boy (Case 8) presented with intractable epilepsy secondary to left parietooccipital transmantle heterotopia extending into the ventricle and abutting the thalamus, immediately adjacent to the posterior limb of the internal capsule. Additionally, the patient presented with a large occipital meningeal cyst (Fig. 3). Following discussion at the multidisciplinary epilepsy meeting, it was decided to proceed with resection of the heterotopia and to perform a left temporal lobectomy. BrainLAB neuronavigation uploaded with preoperative MRI was used to localize the initial site of resection, and ECoG was performed indicating discharge in the left parietooccipital junction adjacent to the anterior margin of the occipital cyst, along the temporal lobe including the left superior

TABLE 3. Descriptive characteristics for patients undergoing iMRI-assisted peri-eloquent resections

\begin{tabular}{|c|c|c|c|c|c|c|c|c|}
\hline $\begin{array}{l}\text { Case } \\
\text { No. }\end{array}$ & $\begin{array}{l}\text { Age (yrs), } \\
\text { Sex }\end{array}$ & $\begin{array}{l}\text { Histopathology } \\
\text { (FCD type) }\end{array}$ & Lesion Location & $\begin{array}{c}\text { Operative } \\
\text { Duration } \\
\text { (mins) }\end{array}$ & $\begin{array}{l}\text { Return to } \\
\text { Surgery } \\
\text { After iMRI }\end{array}$ & $\begin{array}{l}\text { Follow- } \\
\text { Up (mos) }\end{array}$ & $\begin{array}{l}\text { Engel } \\
\text { Class }\end{array}$ & Postop Morbidity \\
\hline 1 & $17, \mathrm{~F}$ & I & Lt temporal & 261 & No & 0.5 & I & No \\
\hline 2 & $6, F$ & IIB & Lt temporoparietal & 382 & Yes & 10.6 & I & No \\
\hline 3 & $1, M$ & NS & Rt parietal & 382 & No & 0.8 & I & No \\
\hline 4 & $3, \mathrm{~F}$ & I & Rt parietal & 482 & Yes & 9.1 & I & Lt hemiparesis \\
\hline 5 & $13, \mathrm{~F}$ & $\| A$ & Rt parietal & 371 & No & 9.8 & I & Altered It lower-extremity sensation \\
\hline 6 & $10, \mathrm{~F}$ & $\| A$ & Lt parietal & 293 & No & 7.3 & I & Rt upper-extremity monoparesis \\
\hline 7 & $7, \mathrm{~F}$ & $\| A$ & Rt insular & 361 & No & 6.9 & III & No \\
\hline 8 & $2, M$ & NS & $\begin{array}{l}\text { Lt parietooccipital transmantle } \\
\text { heterotopia }\end{array}$ & 798 & Yes & 5.7 & $I^{*}$ & No \\
\hline 9 & $8, F$ & $\| A$ & Lt parietal & 514 & Yes & 1.8 & I & Altered rt lower-extremity sensation \\
\hline 10 & $9, \mathrm{M}$ & IIB & Lt frontal & 352 & No & 1.3 & 1 & No \\
\hline 11 & $22, \mathrm{~F}$ & $\| \mathrm{A}$ & Lt frontal & 309 & No & 1.5 & 1 & No \\
\hline
\end{tabular}

\footnotetext{
* Patient underwent repeat iMRI-assisted surgery 1 month postoperatively, achieving GTR and complete seizure freedom (Engel Class I).
} 

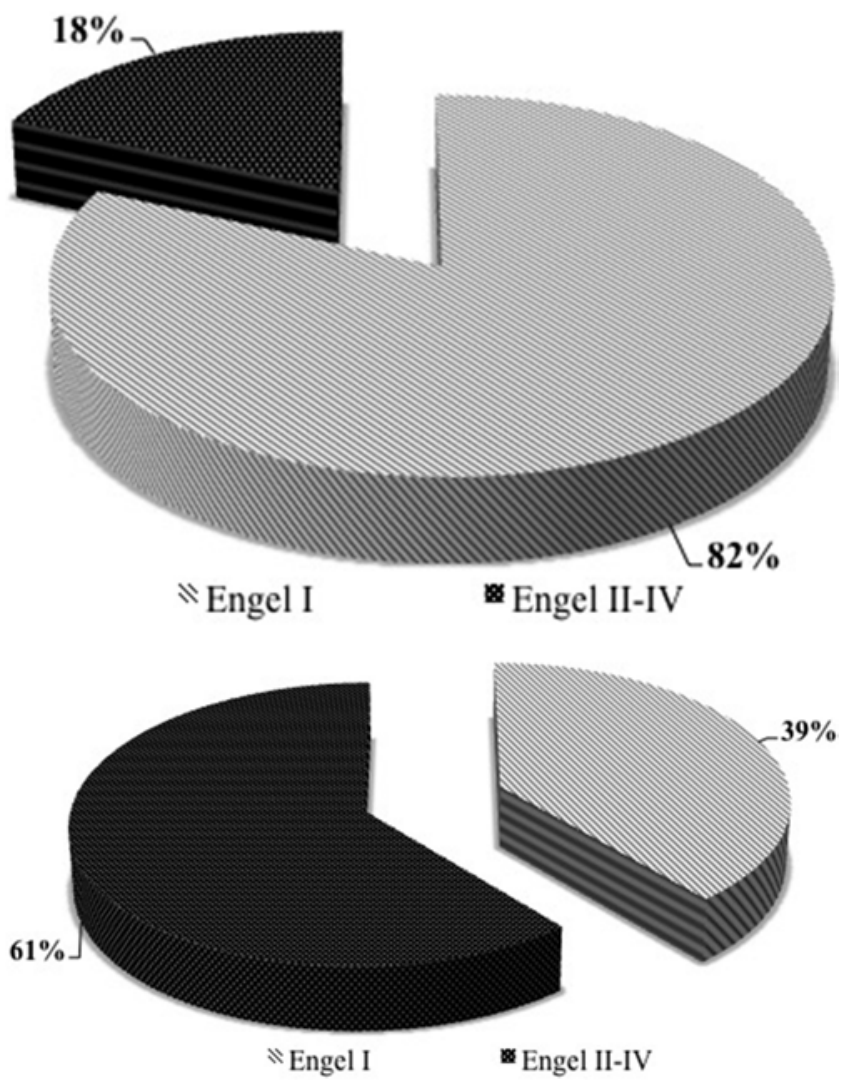

FIG. 1. Pie charts illustrating postoperative seizure freedom at the last postoperative follow-up evaluation in the iMRI-assisted resection cohort (upper) and the conventional resection cohort (lower). temporal gyrus. To avoid the risk of hemiparesis, an approach from a posterior-inferior aspect was undertaken, resecting the dysplasia from its inferior margin medially to the choroidal fissure. Next, resection of the left posterior parietal gyri was carried through to the midline. In the depths of the cortical dissection, heterotopia was identified. It should be noted that at this point SSEPs and MEPs remained stable. However, as the resection continued anteriorly, there was a reduction in evoked potential. At this point, the resection was stopped and the patient prepared for iMRI. Both T1 spoiled gradient recalled echo and T2weighted sequences confirmed residual dysplasia, and the patient was returned to surgery for further resection of the anterolateral margins (Fig. 4). Again, attempted resection was accompanied by decreased MEPs and the procedure was stopped. Postoperatively, the patient saw a reduction in seizures from 9/day to 2/day; however, a decision was made to repeat surgery 1 month later to resect the residual dysplasia. Resection of the deeper aspect of the residual heterotopia was conducted until the atrium of the left lateral ventricle was encountered, then continued laterally and inferiorly. Throughout this point, there were no changes in the MEPs. A complete disconnection of the occipital lobe was then undertaken. Intraoperative T1 spoiled gradient recalled echo, T2-weighted, and DWI images confirmed a complete resection (Fig. 5). Postoperative follow-up at 5 months indicated the patient had achieved complete seizure freedom (Engel Class I).

\section{Discussion}

Resection of FCD localized adjacent to eloquent cortex

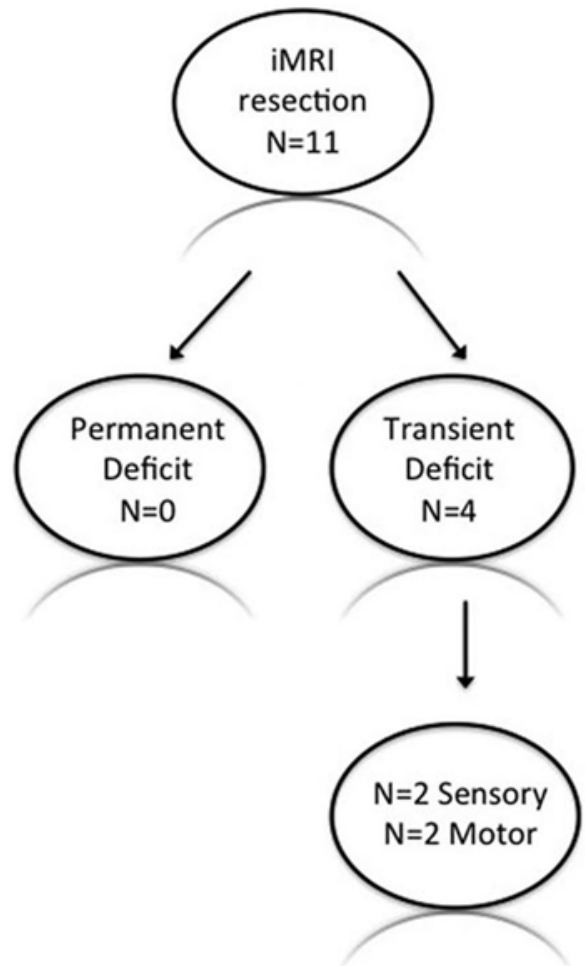

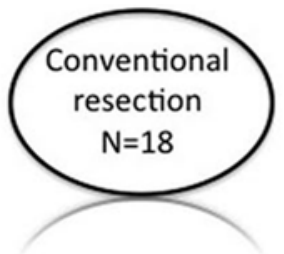

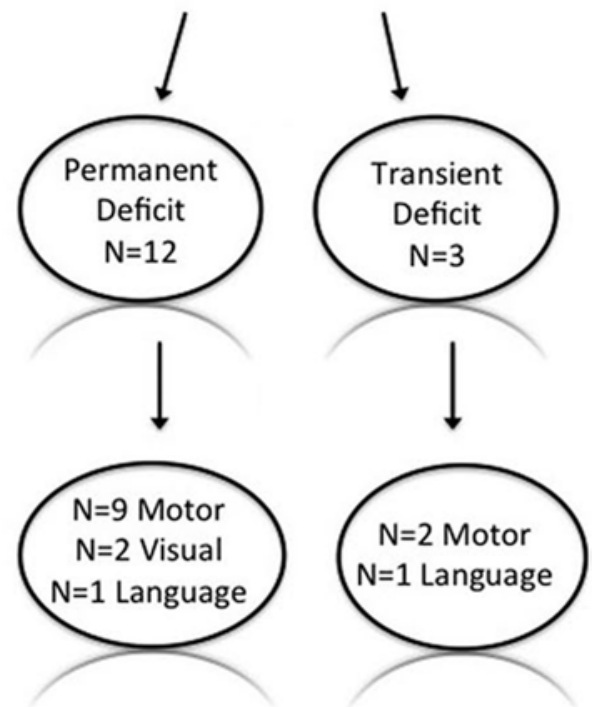

FIG. 2. Postoperative neurological deficits in the iMRI-assisted and conventional resection cohorts. 

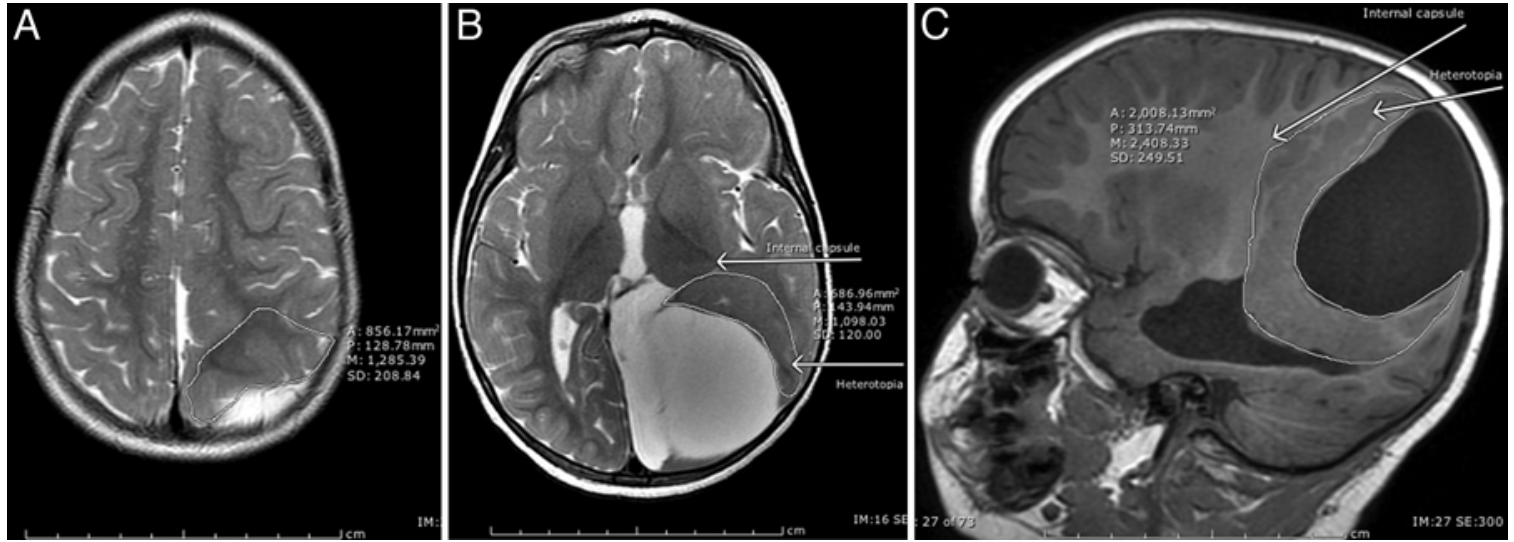

FIG. 3. Case 8. Preoperative MR images. A: Axial T2-weighted image showing left-sided heterotopia (outline) just behind the left central sulcus. B: Axial T2-weighted image showing the deeper aspects of the left transmantle heterotopia just behind the left internal capsule and left thalamus. C: Sagittal T1-weighted image showing left transmantle heterotopia (note a congenital meningeal cyst posterior to the heterotopia).

presents a distinct challenge. A balance must be achieved between achieving the ultimate goal of complete seizure freedom with preservation of important neurological function. We present evidence for the integration of iMRI into preexisting epilepsy protocol techniques, allowing for improved results on both sides of the spectrum: a more complete resection leading to greater rates of seizure freedom, along with decreased rates of resection-induced new neurological deficits.

Intraoperative MRI-assisted surgeries resulted in nearly

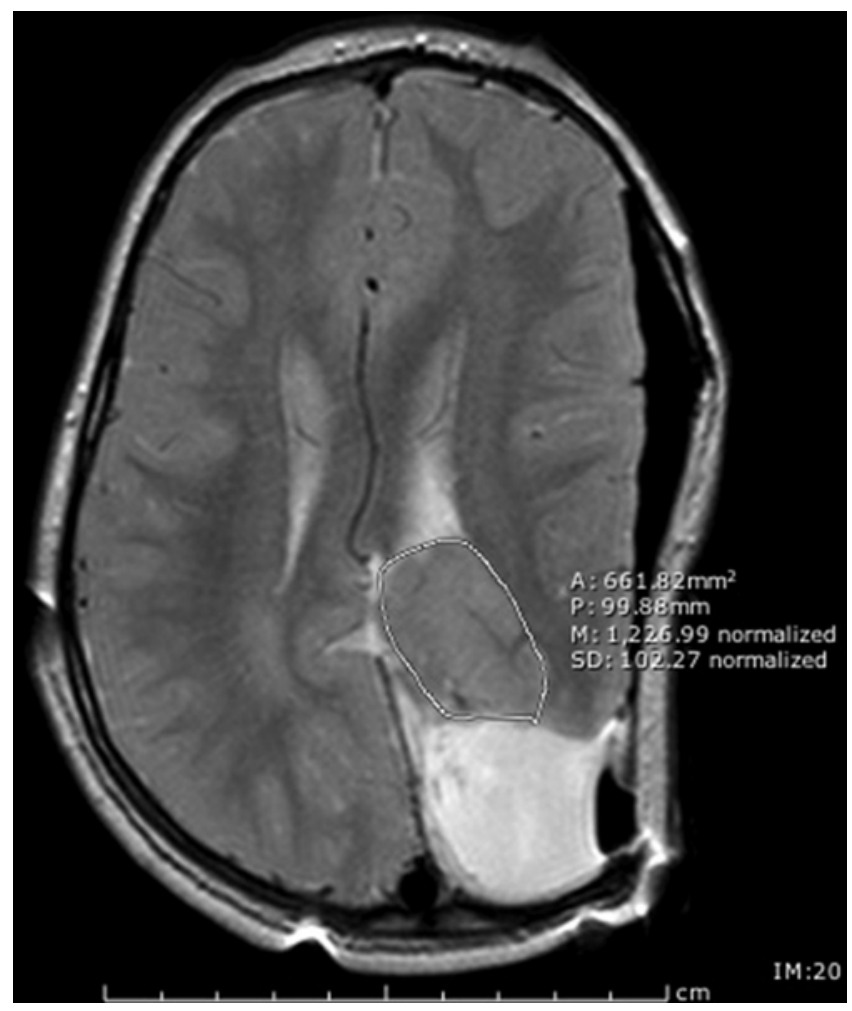

FIG. 4. Case 8. Axial intraoperative T2-weighted MR image at the first surgery showing residual heterotopia (outlined) adjacent to the left internal capsule. Surgery was discontinued at this stage due to changes in MEPs.
$50 \%$ greater rate of GTR than conventional surgeries $(\mathrm{p}=$ 0.02). Furthermore, patients in the iMRI cohort achieved a higher ratio of complete seizure freedom (Engel Class I) than patients in the conventional resection cohort $(\mathrm{p}=$ $0.05)$. Using iMRI in conjunction with functional MRI and diffusion tensor imaging (DTI), Sommer et al. previously reported achieving GTR in all patients and $72 \%$ complete seizure freedom in resection of lesional epilepsy in close proximity to eloquent cortex. ${ }^{32}$ Of note, their cohort consisted of 7 (28\%) of 25 patients with intractable epilepsy secondary to FCD (1 Type IIA and 6 Type IIB), with the remainder of the cases secondary to mixed etiology including posttraumatic glial scars and cavernous hemangiomas. Importantly, surgical outcomes in our study are consistent with those reported by Sommer et al. and considerably improved over conventional resection both in our current study and in the literature. ${ }^{23}$ Prior studies of resection of lesional epilepsy adjacent to regions of eloquent cortex in pediatric patients have reported complete seizure freedom (Engel Class I) rates of $43 \%-64 \%, 1,2,8,24$

Patients in the conventional resection cohort developed postoperative neurological deficits at a higher rate than
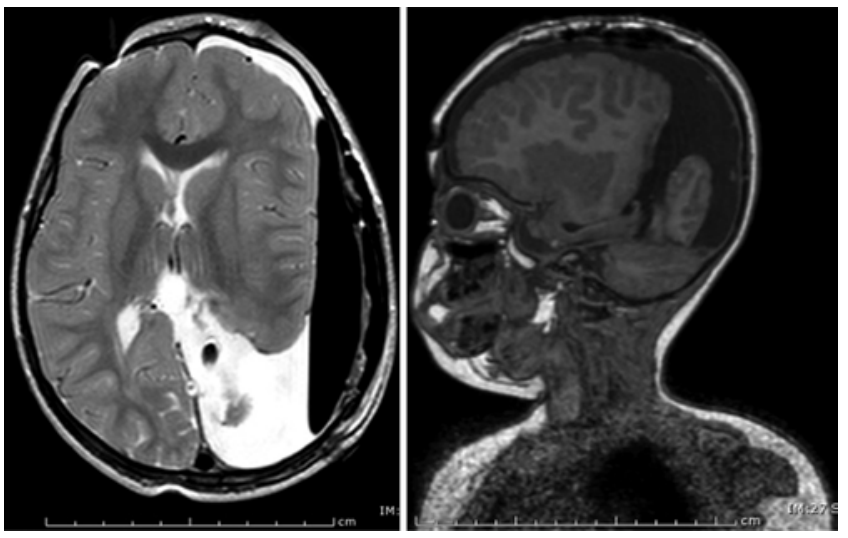

FIG. 5. Case 8. Left: Axial intraoperative T2-weighted MR image at the second surgery showing complete resection of the residual heterotopia. Right: Sagittal intraoperative T2-weighted MR image at the second surgery showing complete resection of the residual heterotopia. 
patients undergoing iMRI-assisted resection $(\mathrm{p}=0.02)$. Again, our reported rates of $36 \%$ transient and no permanent new neurological deficits in the iMRI cohort are consistent with those of Sommer et al., who reported $20 \%$ transient and $12 \%$ permanent neurological deficits in their cohort of iMRI-resected extratemporal epilepsy adjacent to eloquent cortex. ${ }^{32}$ Importantly, this represents a reduction in deficits over conventional resections in our study, and in previous studies that have reported postoperative neurological deficits ranging from $50 \%$ to $83 \% .^{1-3,25}$ Hence, our results demonstrate that iMRI-assisted resections are less prone to incurring neurological deficits. This may be due, in part, to the surgeon undertaking a more conservative extirpation with the knowledge that iMRI may provide a "second chance" to reevaluate the surgical course of action; thus in cases of FCD adjacent to eloquent cortex this minimizes the risk of damage to functional regions and enhances postoperative quality of life.

The most significant factor leading to postoperative seizure freedom in patients with intractable epilepsy secondary to FCD is GTR of the MRI-indicated lesion. . $^{11,12,14,16,27}$ We found that iMRI allowed for the intraoperative ability to examine the extent of resection once the surgeon believed a complete surgery had been accomplished. In cases of MR-indicated residual dysplasia, iMRI became especially critical in enabling the surgeon to account for brain shift (which may distort accurate depiction of eloquent anatomical landmarks on preoperative images) and update neuronavigation for a reevaluation of the resection cavity. In our present study, iMRI impacted the course of surgery in 4 cases, with repeat surgery precluded in 3 of those cases. Conversely, more than one-third of the patients in the conventional resection cohort underwent reoperations for residual dysplasia. In preventing unnecessary reoperations, iMRI acts as a quality control mechanism that both reduces the risk associated with further surgery (a factor that becomes even more substantial in eloquent cortex resections) and has profound implications from a health economics standpoint.

A critique of iMRI utilization has been that it may lead to more aggressive resections of MR-indicated lesions, thus increasing the risk of postoperative morbidity in eloquent cortex surgeries. ${ }^{29}$ As Case 8 illustrates, iMRI seamlessly integrates with intraoperative neurophysiological monitoring (a functional "security margin" that is often more precise than anatomical indication of eloquent cortex alone), thus reducing the risk associated with overly aggressive resection of intraoperative MR-indicated residual lesions. Similar findings have been reported in resection of gliomas localized to eloquent cortex where iMRI in combination with intraoperative neurophysiological monitoring allows for an optimal resection within functional boundaries. ${ }^{30}$ Equally important may be avoiding essential white matter tracts, which has been aided by the advent of DTI. Yet, a known intraoperative challenge of functional MRI with DTI utilization is the effect of brain shift. To counter this, iMRI has been successfully integrated with these imaging techniques in lesional epilepsy surgery.,32 In addition to traditional intraoperative CT and MRI, further technologies such as advanced intraoperative ultrasonography and intraoperative elasticity imaging may better aid in real-time identification of dysplastic lesions and in more accurate depiction of margins. ${ }^{5,18}$ Furthermore, intraoperative ultrasonography has been shown to successfully integrate with functional MRI and DTI to provide an intraoperative update to neuronavigation accounting for brain shift. ${ }^{26}$

While we found iMRI to be a powerful addition to preexisting epilepsy protocol techniques, its resolution is limited to approximately $1 \mathrm{~mm} \cdot{ }^{17}$ In eloquent cortex resections where lesions less than $5 \mathrm{~mm}$ from functional tissue are associated with increased risk of neurological deficit, iMRI resolution may potentially be limited. ${ }^{15}$ Although our study represents one of the larger investigations of lesional resection adjacent to eloquent cortex, due to the relatively small number of cases involving FCD localized to this region, our study is limited by sample size $(n=29)$. Furthermore, while we are among the first to illustrate the capacity of iMRI to aid in FCD eloquent cortex resections, the median follow-up duration for iMRI-assisted resections (5.72 months) is relatively short compared with conventional resections. Of note, however, we found that for the 11 patients who suffered seizure recurrence following conventional FCD resection, the median duration to postoperative seizure recurrence was 2.23 months. Finally, although microsurgery was used in all cases of our study, our results may be confounded by advanced experience over time and in the utilization (or lack thereof) of other neurosurgical assistive technologies. Additionally, differences in surgeons performing the conventional and iMRI-assisted surgeries may present a limitation of operator dependency bias. Furthermore, differences in radiological interpretation from 1.5-T versus 3.0-T MRI may have skewed our results. The current study may benefit from a multiinstitutional study with a larger cohort of patients and longer follow-up duration.

\section{Conclusions}

The results of this study suggest that in comparison with a conventional surgical protocol and technique for resection of FCD localized adjacent to eloquent cortex, the incorporation of iMRI aids in achieving greater rates of GTR and complete seizure freedom. Additionally, iMRIassisted surgeries are associated with reduced rates of neurological deficit in the functional cortex. When paired with neurophysiological monitoring, iMRI may provide additional information to guide intraoperative decision making in regions of eloquent cortex.

\section{References}

1. Behdad A, Limbrick DD Jr, Bertrand ME, Smyth MD: Epilepsy surgery in children with seizures arising from the rolandic cortex. Epilepsia 50:1450-1461, 2009

2. Benifla M, Sala F Jr, Jane J, Otsubo H, Ochi A, Drake J, et al: Neurosurgical management of intractable rolandic epilepsy in children: role of resection in eloquent cortex. Clinical article. J Neurosurg Pediatr 4:199-216, 2009

3. Buchfelder M, Fahlbusch R, Ganslandt O, Stefan H, Nimsky C: Use of intraoperative magnetic resonance imaging in tailored temporal lobe surgeries for epilepsy. Epilepsia 43:864-873, 2002

4. Buchfelder M, Ganslandt O, Fahlbusch R, Nimsky C: Intra- 
operative magnetic resonance imaging in epilepsy surgery. $\mathbf{J}$ Magn Reson Imaging 12:547-555, 2000

5. Chan HW, Pressler R, Uff C, Gunny R, St Piers K, Cross H, et al: A novel technique of detecting MRI-negative lesion in focal symptomatic epilepsy: intraoperative ShearWave elastography. Epilepsia 55:e30-e33, 2014

6. Cohen-Gadol AA, Ozduman K, Bronen RA, Kim JH, Spencer DD: Long-term outcome after epilepsy surgery for focal cortical dysplasia. J Neurosurg 101:55-65, 2004

7. Cui ZQ, Ling ZP, Song HF, Hu S, Sun GC, Chen XL, et al: Combining pyramidal tract mapping, microscopic-based neuronavigation, and intraoperative magnetic resonance imaging improves outcome of epilepsy foci resection in the sensorimotor cortex. Turk Neurosurg 24:538-545, 2014

8. de Oliveira RS, Santos MV, Terra VC, Sakamoto AC, Machado HR: Tailored resections for intractable rolandic cortex epilepsy in children: a single-center experience with 48 consecutive cases. Childs Nerv Syst 27:779-785, 2011

9. Delev D, Send K, Wagner J, von Lehe M, Ormond DR, Schramm J, et al: Epilepsy surgery of the rolandic and immediate perirolandic cortex: surgical outcome and prognostic factors. Epilepsia 55:1585-1593, 2014

10. Engel J, Van Ness PC, Rasmussen TB, Ojemann LM: Outcome with respect to epileptic seizures, in Engel J (ed): Surgical Treatment of the Epilepsies, ed 2. New York: Raven Press, 1993, 609-621

11. Fauser S, Bast T, Altenmüller DM, Schulte-Mönting J, Strobl $\mathrm{K}$, Steinhoff BJ, et al: Factors influencing surgical outcome in patients with focal cortical dysplasia. J Neurol Neurosurg Psychiatry 79:103-105, 2008

12. Hader WJ, Mackay M, Otsubo H, Chitoku S, Weiss S, Becker L, et al: Cortical dysplastic lesions in children with intractable epilepsy: role of complete resection. J Neurosurg 100 (2 Suppl Pediatrics):110-117, 2004

13. Hatiboglu MA, Weinberg JS, Suki D, Tummala S, Rao G, Sawaya R, et al: Utilization of intraoperative motor mapping in glioma surgery with high-field intraoperative magnetic resonance imaging. Stereotact Funct Neurosurg 88:345352,2010

14. Kim DW, Lee SK, Chu K, Park KI, Lee SY, Lee CH, et al: Predictors of surgical outcome and pathologic considerations in focal cortical dysplasia. Neurology 72:211-216, 2009

15. Krishnan R, Raabe A, Hattingen E, Szelényi A, Yahya H, Hermann E, et al: Functional magnetic resonance imagingintegrated neuronavigation: Correlation between lesion-tomotor cortex distance and outcome. Neurosurgery 55:904915, 2004

16. Krsek P, Maton B, Jayakar P, Dean P, Korman B, Rey G, et al: Incomplete resection of focal cortical dysplasia is the main predictor of poor postsurgical outcome. Neurology 72:217-223, 2009

17. Lüsebrink F, Wollrab A, Speck O: Cortical thickness determination of the human brain using high resolution $3 \mathrm{~T}$ and $7 \mathrm{~T}$ MRI data. Neuroimage 70:122-131, 2013

18. Miller D, Knake S, Bauer S, Krakow K, Pagenstecher A, Sure $\mathrm{U}$, et al: Intraoperative ultrasound to define focal cortical dysplasia in epilepsy surgery. Epilepsia 49:156-158, 2008

19. Nimsky C, Ganslandt O, Buchfelder M, Fahlbusch R: Intraoperative visualization for resection of gliomas: the role of functional neuronavigation and intraoperative $1.5 \mathrm{~T}$ MRI. Neurol Res 28:482-487, 2006

20. Nimsky C, Ganslandt O, Cerny S, Hastreiter P, Greiner G, Fahlbusch R: Quantification of, visualization of, and compensation for brain shift using intraoperative magnetic resonance imaging. Neurosurgery 47:1070-1080, 2000

21. Nimsky C, Ganslandt O, Gralla J, Buchfelder M, Fahlbusch
$\mathrm{R}$ : Intraoperative low-field magnetic resonance imaging in pediatric neurosurgery. Pediatr Neurosurg 38:83-89, 2003

22. Nimsky C, Ganslandt O, Hastreiter P, Fahlbusch R: Intraoperative compensation for brain shift. Surg Neurol 56:357365,2001

23. Oluigbo CO, Wang J, Whitehead MT, Magge S, Myseros JS, Yaun A, et al: The influence of lesion volume, perilesion resection volume, and completeness of resection on seizure outcome after resective epilepsy surgery for cortical dysplasia in children. J Neurosurg Pediatr 15:644-650, 2015

24. Otsubo H, Chitoku S, Ochi A, Jay V, Rutka JT, Smith ML, et al: Malignant rolandic-sylvian epilepsy in children: diagnosis, treatment, and outcomes. Neurology 57:590-596, 2001

25. Pondal-Sordo M, Diosy D, Téllez-Zenteno JF, Girvin JP, Wiebe S: Epilepsy surgery involving the sensory-motor cortex. Brain 129:3307-3314, 2006

26. Rasmussen IA Jr, Lindseth F, Rygh OM, Berntsen EM, Selbekk T, Xu J, et al: Functional neuronavigation combined with intra-operative 3D ultrasound: initial experiences during surgical resections close to eloquent brain areas and future directions in automatic brain shift compensation of preoperative data. Acta Neurochir (Wien) 149:365-378, 2007

27. Rowland NC, Englot DJ, Cage TA, Sughrue ME, Barbaro NM, Chang EF: A meta-analysis of predictors of seizure freedom in the surgical management of focal cortical dysplasia. J Neurosurg 116:1035-1041, 2012

28. Sarkis RA, Jehi LE, Bingaman WE, Najm IM: Surgical outcome following resection of rolandic focal cortical dysplasia. Epilepsy Res 90:240-247, 2010

29. Senft C: Glioma surgery: intraoperative low field magnetic resonance imaging, in Hayat MA (ed): Tumors of the Central Nervous System. Gliomas: Glioblastoma. Dordrecht: Springer, 2011, Vol 2, pp 180-188

30. Senft C, Forster MT, Bink A, Mittelbronn M, Franz K, Seifert V, et al: Optimizing the extent of resection in eloquently located gliomas by combining intraoperative MRI guidance with intraoperative neurophysiological monitoring. J Neurooncol 109:81-90, 2012

31. Senft C, Franz K, Blasel S, Oszvald A, Rathert J, Seifert V, et al: Influence of iMRI-guidance on the extent of resection and survival of patients with glioblastoma multiforme. Technol Cancer Res Treat 9:339-346, 2010

32. Sommer B, Grummich P, Coras R, Kasper BS, Blumcke I, Hamer HM, et al: Integration of functional neuronavigation and intraoperative MRI in surgery for drug-resistant extratemporal epilepsy close to eloquent brain areas. Neurosurg Focus 34(4):E4, 2013

\section{Disclosures}

The authors report no conflict of interest concerning the materials or methods used in this study or the findings specified in this paper.

\section{Author Contributions}

Conception and design: Oluigbo, Sacino, Gaillard. Acquisition of data: Oluigbo, Sacino. Analysis and interpretation of data: Oluigbo, Sacino. Drafting the article: Oluigbo, Sacino. Critically revising the article: Oluigbo, Sacino. Reviewed submitted version of manuscript: all authors. Statistical analysis: Oluigbo, Sacino. Study supervision: Oluigbo.

\section{Correspondence}

Chima O. Oluigbo, Departments of Neurosurgery, Children's National Medical Center, 111 Michigan Ave. NW, Washington, DC 20010. email: coluigbo@cnmc.org. 